\title{
Kaluza-Klein Dark Matter
}

\author{
Hsin-Chia Cheng, ${ }_{1}^{1}$ Jonathan L. Feng, ${ }^{2}$ and Konstantin T. Matchev ${ }^{3,4}$ \\ ${ }^{1}$ Enrico Fermi Institute, The University of Chicago, Chicago, IL 60637, USA \\ ${ }^{2}$ Department of Physics and Astronomy, University of California, Irvine, CA 92697, USA \\ ${ }^{3}$ Department of Physics, University of Florida, Gainesville, FL 32611, USA \\ ${ }^{4}$ Theory Division, CERN, CH-1211 Geneva, Switzerland
}

\begin{abstract}
We propose that cold dark matter is made of Kaluza-Klein particles and explore avenues for its detection. The lightest Kaluza-Klein state is an excellent dark matter candidate if standard model particles propagate in extra dimensions and Kaluza-Klein parity is conserved. We consider KaluzaKlein gauge bosons. In sharp contrast to the case of supersymmetric dark matter, these annihilate to hard positrons, neutrinos and photons with unsuppressed rates. Direct detection signals are also promising. These conclusions are generic to bosonic dark matter candidates.
\end{abstract}

PACS numbers: $12.60 .-\mathrm{i}, 11.10 . \mathrm{Kk}, 95.35 .+\mathrm{d}$

The identity of dark matter is currently among the most profound mysteries in particle physics, astrophysics, and cosmology. Recent data from supernovae luminosities, cosmic microwave anisotropies, and galactic rotation curves all point consistently to the existence of dark matter with mass density $\Omega \approx 0.3$ relative to the critical density. At the same time, all known particles are excluded as dark matter candidates, making the dark matter problem the most pressing phenomenological motivation for particles and interactions beyond the standard model.

Among the myriad options, the possibility of particle dark matter with weak interactions and weak-scale mass is particularly tantalizing. Puzzles concerning electroweak symmetry breaking suggest that such particles exist, and, if stable, their thermal relic density is generically in the desired range. Among these candidates, neutralinos in supersymmetric theories are by far the most widely studied. Neutralinos have spin $1 / 2$ and are their own anti-particles; that is, they are Majorana fermions. They may be detected directly through scattering in detectors, or indirectly through the decay products that result when neutralinos annihilate in pairs. For indirect detection, however, the Majorana nature of neutralinos implies that annihilation is chirality-suppressed, leading to soft secondary positrons, photons, and neutrinos, and considerably diminishing prospects for discovery.

Here we study a specific example of a generic alternative: bosonic cold dark matter. If particles propagate in extra spacetime dimensions, they will have an infinite tower of partner states with identical quantum numbers, as noted long ago by Kaluza and Klein [1]. We consider the case of universal extra dimensions (UED) [2], in which all standard model particles propagate. Such models provide, in the form of stable Kaluza-Klein (KK) partners, the only specific dark matter candidate to emerge from theories with extra dimensions [3, 4, 5]. KK dark matter generically has the desired relic density [6, 7. Here we explore for the first time the prospects for its detection.

For concreteness, we consider the simplest UED model, with one extra dimension of size $R \sim \mathrm{TeV}^{-1}$ compactified on an $S^{1} / Z_{2}$ orbifold. At the lowest order, the KK masses are simply the momenta along the extra dimension and are quantized in units of $1 / R$. The degeneracy at each KK level is lifted by radiative corrections and boundary terms 四. The boundaries also break momentum conservation in the extra dimension down to a $Z_{2}$ parity, under which KK modes with odd KK number are charged. This KK-parity corresponds to the symmetry of reflection about the midpoint in the extra dimension; it is anomaly-free and not violated by quantum gravity effects. KK-parity conservation implies that the lightest KK particle is stable. KK partners of electroweak gauge bosons and neutrinos are then all possible dark matter candidates. We consider $B^{1}$, the first KK mode of the hypercharge gauge boson, which at one-loop is naturally the lightest KK mass eigenstate in minimal models 沺, 5 .

In this UED scenario, constraints from precision data require only $1 / R \gtrsim 300 \mathrm{GeV}$ [2]. Collider searches are also quite challenging: the Tevatron Run II may probe slightly beyond this bound and the LHC may reach $1 / R \sim 1.5 \mathrm{TeV}[5]$. Dark matter searches provide another possibility for probing these models and differentiating them from other new physics.

For a given KK spectrum, the $B^{1}$ thermal relic density may be accurately determined [6]. $B^{1} \mathrm{~s}$ annihilate effectively through $s$-wave processes, unlike neutralinos, and so the desired thermal relic density is obtained for higher masses than typical for neutralinos. If $B^{1} \mathrm{~s}$ are the only KK modes with significant abundance at the freezeout temperature, the desired relic density is found for $m_{B^{1}} \approx 1 \mathrm{TeV}$. However, many other KK states may be closely degenerate with $B^{1}$, and their presence at freezeout will modify this conclusion. KK quarks and gluons annihilate with much larger cross sections through strong interactions, and so increase the predicted $m_{B^{1}}$. On the other hand, degenerate KK leptons lower the average annihilation cross section and require lower $m_{B^{1}}$. In addition to the cosmological assumptions present in all relic density calculations, the $B^{1}$ relic density is therefore 
rather model-dependent, with the optimal $m_{B^{1}}$ ranging from several hundred $\mathrm{GeV}$ to a few $\mathrm{TeV}$, depending sensitively on the KK spectrum. Here we study the prospects for detection in a model-independent way by considering $m_{B^{1}}$ as a free parameter in the appropriate range.

We first consider the direct detection of $B^{1}$ dark matter. Dark matter particles are currently non-relativistic, with velocity $v \sim 10^{-3}$. For weak-scale dark matter, the recoil energy from scattering off nuclei is $\sim 0.1 \mathrm{MeV}$, and far less for scattering off electrons. We therefore consider elastic scattering off nucleons and nuclei.

At the quark level, $B^{1}$ scattering takes place through KK quarks, with amplitude $\mathcal{M}_{q}^{q^{1}}=\mathcal{M}_{q_{L}}^{q^{1}}+\mathcal{M}_{q_{R}}^{q^{1}}$, where

$$
\begin{aligned}
& \mathcal{M}_{q_{i}}^{q^{1}}=-i \frac{e^{2}}{\cos ^{2} \theta_{W}} Y_{q_{i}}^{2} \varepsilon_{\mu}^{*}\left(p_{3}\right) \varepsilon_{\nu}\left(p_{1}\right) \times \\
& \bar{u}\left(p_{4}\right)\left[\frac{\gamma^{\mu} \not k_{1} \gamma^{\nu}}{k_{1}^{2}-m_{q_{i}^{1}}^{2}}+\frac{\gamma^{\nu} \not k_{2} \gamma^{\mu}}{k_{2}^{2}-m_{q_{i}^{1}}^{2}}\right] P_{i} u\left(p_{2}\right),
\end{aligned}
$$

$Y=Q-I$ is hypercharge, $k_{1}=p_{1}+p_{2}$, and $k_{2}=p_{2}-p_{3}$; and through Higgs exchange, with amplitude

$$
\mathcal{M}_{q}^{h}=i \frac{e^{2}}{2 \cos ^{2} \theta_{W}} \frac{m_{f}}{k_{3}^{2}-m_{h}^{2}} \varepsilon_{\mu}^{*}\left(p_{3}\right) \varepsilon^{\mu}\left(p_{1}\right) \bar{u}\left(p_{4}\right) u\left(p_{2}\right),
$$

where $k_{3}=p_{1}-p_{3}$. In the extreme non-relativistic limit, $p_{1}=p_{3}=\left(m_{B^{1}}, \mathbf{0}\right)$, and expanding to linear order in $p_{2}=\left(E_{q}, \boldsymbol{p}_{q}\right)$, these amplitudes then reduce to

$$
\begin{aligned}
\mathcal{M}_{q}^{q^{1}} \approx & \alpha_{q} \varepsilon_{\mu}^{*}\left(p_{3}\right) \varepsilon_{\nu}\left(p_{1}\right) \varepsilon^{0 \mu \nu \rho} \xi_{4}^{\dagger} \frac{\sigma_{\rho}}{2} \xi_{2} \\
& -i \beta_{q} \varepsilon_{\mu}^{*}\left(p_{3}\right) \varepsilon^{\mu}\left(p_{1}\right) \xi_{4}^{\dagger} \xi_{2} \\
\mathcal{M}_{q}^{h} \approx & -i \gamma_{q} \varepsilon_{\mu}^{*}\left(p_{3}\right) \varepsilon^{\mu}\left(p_{1}\right) \xi_{4}^{\dagger} \xi_{2}
\end{aligned}
$$

where $\xi_{4}$ and $\xi_{2}$ are two-component spinors, and

$$
\begin{aligned}
\alpha_{q} & =\frac{2 e^{2}}{\cos ^{2} \theta_{W}}\left[\frac{Y_{q_{L}}^{2} m_{B^{1}}}{m_{q_{L}^{1}}^{2}-m_{B^{1}}^{2}}+(L \rightarrow R)\right] \\
\beta_{q} & =E_{q} \frac{e^{2}}{\cos ^{2} \theta_{W}}\left[Y_{q_{L}}^{2} \frac{m_{B^{1}}^{2}+m_{q_{L}^{1}}^{2}}{\left(m_{q_{L}^{1}}^{2}-m_{B^{1}}^{2}\right)^{2}}+(L \rightarrow R)\right] \\
\gamma_{q} & =m_{q} \frac{e^{2}}{2 \cos ^{2} \theta_{W}} \frac{1}{m_{h}^{2}} .
\end{aligned}
$$

The interactions divide into spin-dependent and spinindependent parts [8]. Higgs exchange contributes to scalar couplings, while $q^{1}$ exchange contributes to both. Note that the two contributions to scalar interactions interfere constructively; barring extremely heavy KK masses, there is an inescapable lower bound on both spindependent and scalar cross sections.

The spin-dependent coupling is $\alpha_{q} \boldsymbol{S}_{B^{1}} \cdot \boldsymbol{S}_{q}$, where $\boldsymbol{S}_{B^{1}}$ and $\boldsymbol{S}_{q}$ are spin operators. We must evaluate this matrix element between nucleon or nucleus bound states. By the Wigner-Eckart theorem, we may replace $\boldsymbol{S}_{q}$ by $\lambda_{q} \boldsymbol{J}_{N}$, where $\boldsymbol{J}_{N}$ is the nucleon or nuclear spin operator. The constant of proportionality is

$$
\lambda_{q}=\Delta_{q}^{p}\left\langle S_{p}\right\rangle / J_{N}+\Delta_{q}^{n}\left\langle S_{n}\right\rangle / J_{N} .
$$

$\Delta_{q}^{p, n}$ is given by $\left\langle p, n\left|\boldsymbol{S}_{q}^{\mu}\right| p, n\right\rangle \equiv \Delta_{q}^{p, n} \boldsymbol{S}_{p, n}^{\mu}$ and is the fraction of the nucleon spin carried by quark $q$. A recent analysis finds $\Delta_{u}^{p}=\Delta_{d}^{n}=0.78 \pm 0.02, \Delta_{d}^{p}=$ $\Delta_{u}^{n}=-0.48 \pm 0.02$, and $\Delta_{s}^{p}=\Delta_{s}^{n}=-0.15 \pm 0.02$ [9]. $\left\langle S_{p, n}\right\rangle / J_{N} \equiv\left\langle N\left|S_{p, n}\right| N\right\rangle / J_{N}$ is the fraction of the total nuclear spin $J_{N}$ that is carried by the spin of protons or neutrons. For scattering off protons and neutrons, $\lambda_{q}$ reduces to $\Delta_{q}^{p}$ and $\Delta_{q}^{n}$, respectively.

The spin-dependent cross section is $m_{N}^{2} /\left[4 \pi\left(m_{B^{1}}+\right.\right.$ $\left.\left.m_{N}\right)^{2}\right]\left\langle|\mathcal{M}|^{2}\right\rangle$, where $\mathcal{M}=\sum_{q} \mathcal{M}_{q}$ and \langle\rangle denotes an average over initial polarizations and sum over final polarizations. Using $\left\langle\left(\boldsymbol{S}_{B^{1}} \cdot \boldsymbol{J}_{N}\right)^{2}\right\rangle=\frac{2}{3} J_{N}\left(J_{N}+1\right)$, we find

$$
\sigma_{\text {spin }}=\frac{1}{6 \pi} \frac{m_{N}^{2}}{\left(m_{B^{1}}+m_{N}\right)^{2}} J_{N}\left(J_{N}+1\right)\left[\sum_{u, d, s} \alpha_{q} \lambda_{q}\right]^{2},
$$

where $\alpha_{q}$ and $\lambda_{q}$ are given in Eqs. (5) and (8).

The spin-independent cross section is

$$
\sigma_{\text {scalar }}=\frac{m_{N}^{2}}{4 \pi\left(m_{B^{1}}+m_{N}\right)^{2}}\left[Z f_{p}+(A-Z) f_{n}\right]^{2},
$$

where $Z$ and $A$ are nuclear charge and atomic number,

$$
f_{p}=\sum_{u, d, s}\left(\beta_{q}+\gamma_{q}\right)\langle p|\bar{q} q| p\rangle=\sum_{u, d, s} \frac{\beta_{q}+\gamma_{q}}{m_{q}} m_{p} f_{T_{q}}^{p},
$$

and similarly for $f_{n}$. We take $f_{T_{u}}^{p}=0.020 \pm 0.004, f_{T_{d}}^{p}=$ $0.026 \pm 0.005, f_{T_{u}}^{n}=0.014 \pm 0.003, f_{T_{d}}^{n}=0.036 \pm 0.008$, and $f_{T_{s}}^{p, n}=0.118 \pm 0.062$ [10]. $E_{q}$ of Eq. (6) is the energy of a bound quark and is rather ill-defined. In evaluating Eq. (11), we conservatively replace $E_{q}$ by the current mass $m_{q}$. We also neglect couplings to gluons mediated by heavy quark loops; a detailed loop-level analysis along the lines of Refs. [11, 12] for neutralinos is in progress [13. Given the constructive interference noted above, these contributions can only increase the cross section.

We present both spin-independent and spin-dependent cross sections in Fig. 11. We assume that all first level KK quarks are degenerate with mass $m_{q^{1}}$. Proton cross sections are given; neutron cross sections are similar for spin-dependent interactions and almost identical for scalar cross sections. The cross sections are large for low $m_{B^{1}}$. They are also strikingly enhanced by $r^{-2}$ for small $r \equiv\left(m_{q^{1}}-m_{B^{1}}\right) / m_{B^{1}}$ when scattering takes place near an $s$-channel pole. Such degeneracy is unmotivated in general, but is natural for UED models, where all KK particles are highly degenerate at tree-level.

Projected sensitivities of near future experiments are also shown in Fig. 1. For scattering off individual nucleons, scalar cross sections are suppressed relative to spin-dependent ones by $\sim m_{p} / m_{B^{1}}$. However, this effect 


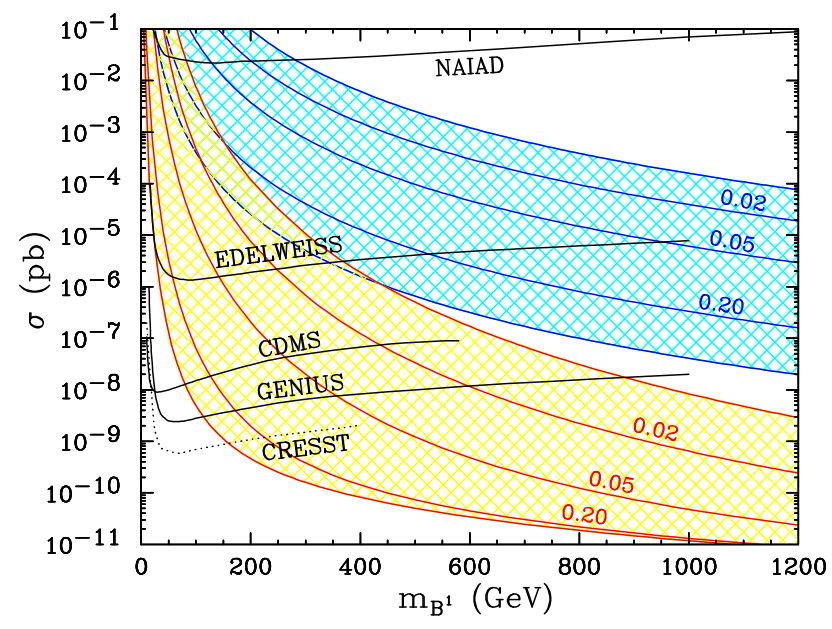

FIG. 1: Predicted spin-dependent proton cross sections (darkshaded, blue), along with the projected sensitivity of a 100 kg NAIAD array [14; and predicted spin-independent proton cross sections (light-shaded, red), along with the current EDELWEISS sensitivity [15], and projected sensitivities of CDMS [16], GENIUS [17, and CRESST [18]. (The CRESST projection is long-term and conditional upon increased exposure and improved background rejection.) The predictions are for $m_{h}=120 \mathrm{GeV}$ and $0.01 \leq r=\left(m_{q^{1}}-m_{B^{1}}\right) / m_{B^{1}} \leq 0.5$, with contours for specific intermediate $r$ labeled.

is compensated in large nuclei where spin-independent rates are enhanced by $\sim A^{2}$. In the case of bosonic KK dark matter, the latter effect dominates, and the spinindependent experiments have the best prospects for detection, with sensitivity to $m_{B^{1}}$ far above current limits.

Dark matter may also be detected when it annihilates in the galactic halo, leading to positron excesses in spacebased and balloon experiments. The positron flux is [19]

$$
\frac{d \Phi_{e^{+}}}{d \Omega d E}=\frac{\rho^{2}}{m_{B^{1}}^{2}} \sum_{i}\left\langle\sigma_{i} v\right\rangle B_{e^{+}}^{i} \int d E_{0} f_{i}\left(E_{0}\right) G\left(E_{0}, E\right)
$$

where $\rho$ is the local dark matter mass density, the sum is over all annihilation channels $i$, and $B_{e^{+}}^{i}$ is the $e^{+}$ branching fraction in channel $i$. The initial positron energy distribution is given by $f\left(E_{0}\right)$, and the Green function $G\left(E_{0}, E\right)$ propagates positrons in the galaxy.

Several channels contribute to the positron flux. Here we focus on the narrow peak of primary positrons from direct $B^{1} B^{1} \rightarrow e^{+} e^{-}$annihilation. (Annihilation to muons, taus and heavy quarks also yield positrons through cascade decays, but with relatively soft and smeared spectra.) In this case, the source is monoenergetic, and Eq. (12) simplifies to

$$
\begin{aligned}
& \frac{d \Phi_{e^{+}}}{d \Omega d E}=2.7 \times 10^{-8} \mathrm{~cm}^{-2} \mathrm{~s}^{-1} \mathrm{sr}^{-1} \mathrm{GeV}^{-1} \frac{\left\langle\sigma_{e e} v\right\rangle}{\mathrm{pb}} \\
& \quad \times\left[\frac{\rho}{0.3 \mathrm{GeV} / \mathrm{cm}^{3}}\right]^{2}\left[\frac{1 \mathrm{TeV}}{m_{B^{1}}}\right]^{2} g\left(1, \frac{E}{m_{B^{1}}}\right),
\end{aligned}
$$

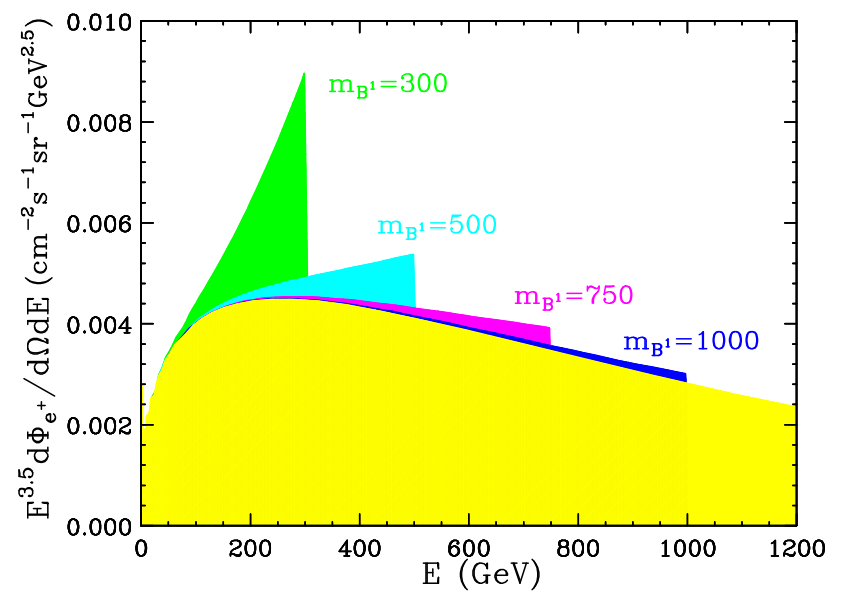

FIG. 2: Predicted positron signals (dark shaded) above background (light shaded) as a function of positron energy for $m_{B^{1}}=m_{e_{L}^{1}}=m_{e_{R}^{1}}=100,500,750$, and $1000 \mathrm{GeV}$.

where the annihilation cross section is

$$
\left\langle\sigma_{e e} v\right\rangle=\frac{e^{4}}{9 \pi \cos ^{4} \theta_{W}}\left[\frac{Y_{e_{L}^{1}}^{4}}{m_{B^{1}}^{2}+m_{e_{L}^{1}}^{2}}+(L \rightarrow R)\right],
$$

and the reduced Green function $g$ is as in Ref. [20].

Positron spectra and an estimated background (model C from Ref. [19) are given in Fig. 2. The sharp peak at $E_{e^{+}}=m_{B^{1}}$ is spectacular - while propagation broadens the spectrum, the mono-energetic source remains evident. This feature is extremely valuable, as the background, although resulting from many sources, should be smooth. Maximal $E_{e^{+}}$also enhances detectability since the background drops rapidly with energy. Both of these virtues are absent for neutralinos, where Majorana-ness implies helicity-suppressed annihilation amplitudes, and positrons are produced only in cascades, leading to soft, smooth spectra 21]. A peak in the $e^{+}$spectrum will not only be a smoking gun for $B^{1}$ dark matter, it will also exclude neutralinos as the source.

Of the many positron experiments, the most promising is AMS [22], the anti-matter detector to be placed on the International Space Station. AMS will distinguish positrons from electrons even at $1 \mathrm{TeV}$ energies [23]. With aperture $6500 \mathrm{~cm}^{2} \mathrm{sr}$ and a runtime of 3 years, AMS will detect $\sim 1000$ positrons with energy above $500 \mathrm{GeV}$, and may detect a positron peak from $B^{1}$ dark matter.

Photons from dark matter annihilation in the center of the galaxy also provide an indirect signal. The line signal from $B^{1} B^{1} \rightarrow \gamma \gamma$ is loop-suppressed, and so we consider continuum photon signals. The integrated photon flux above some photon energy threshold $E_{t h}$ is 20]

$$
\begin{gathered}
\Phi_{\gamma}\left(E_{t h}\right)=5.6 \times 10^{-12} \mathrm{~cm}^{-2} \mathrm{~s}^{-1} \bar{J}(\Delta \Omega) \Delta \Omega \\
\times\left[\frac{1 \mathrm{TeV}}{m_{B^{1}}}\right]^{2} \sum_{q} \frac{\left\langle\sigma_{q q} v\right\rangle}{\mathrm{pb}} \int_{E_{t h}}^{m_{B^{1}}} d E \frac{d N_{\gamma}^{q}}{d E},
\end{gathered}
$$




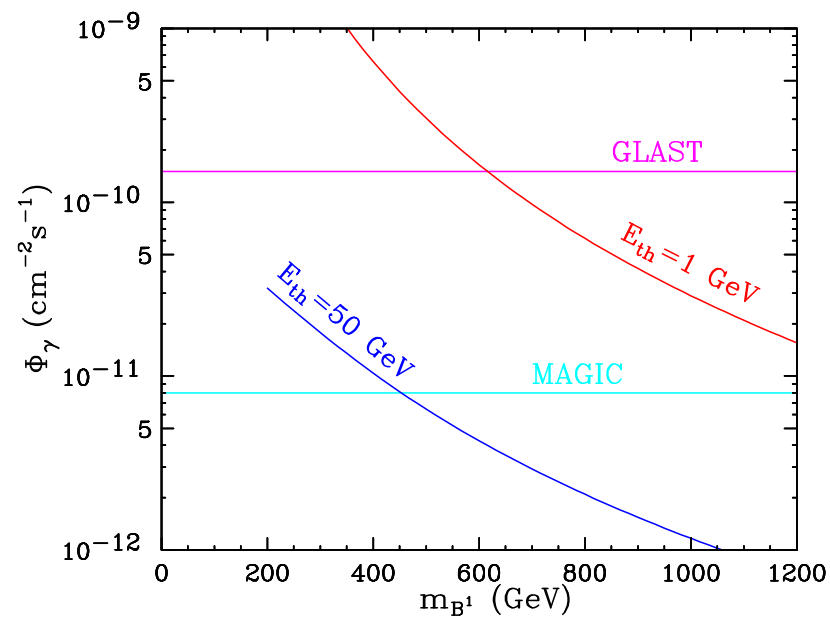

FIG. 3: Integrated photon flux as a function of $m_{B^{1}}$ for energy thresholds of 1 and $50 \mathrm{GeV}$. Projected sensitivities for GLAST and MAGIC are also shown.

where the sum is over all quark pair annihilation channels (with cross sections similar to Eq. (14)), and $d N_{\gamma}^{q} / d E$ is the differential gamma ray multiplicity for channel $q q$. The hardest spectra result from fragmentation of light quarks 24, and so the lack of chirality suppression again gives a relative enhancement over neutralinos. $\Delta \Omega$ is the ang of fict of of a and $\bar{J}$ is a nneasure of the euspiness of the galactic halo density profile. There is a great deal of uncertainty in $\bar{J}$, with possible values in the range 3 to $10^{5}$. We choose $\Delta \Omega=10^{-3}$ and a moderate value of $\bar{J}=500$.

Integrated photon fluxes are given in Fig. 3 for two representative $E_{t h}: 1 \mathrm{GeV}$, accessible to space-based detectors, and $50 \mathrm{GeV}$, characteristic of ground-based telescopes. Estimated sensitivities for two of the more promising experiments, GLAST [25] and MAGIC [26, are also shown. We find that photon excesses are detectable for $m_{B^{1}} \lesssim 600 \mathrm{GeV}$. Note that these signals may be greatly enhanced for clumpy halos with large $\bar{J}$.

Finally, high-energy neutrinos from annihilating dark matter trapped in the core of the Sun or the Earth, can be detected through their charged-current conversion to muons. Unlike the case in supersymmetry, $B^{1} \mathrm{~s}$ can annihilate directly to neutrinos, with branching ratio $\approx 1.2 \%$. Secondary neutrinos may also result from final states with heavy quarks, charged leptons, or Higgs bosons. Considering primary neutrinos and those from tau decays from the Sun (which is typically full, with capture and annihilation in equilibrium), we find that, for $r=0.5$ (0.02), next generation neutrino telescopes like AMANDA, NESTOR and ANTARES will probe $m_{B^{1}}$ up to $200 \mathrm{GeV}(600 \mathrm{GeV})$ and IceCube will be sensitive to $m_{B^{1}}=400 \mathrm{GeV}(1400 \mathrm{GeV})$ [13].

In conclusion, we find excellent prospects for KK dark matter detection. The elastic scattering cross sections are enhanced near $s$-channel KK resonances, providing good chances for direct detection. In addition, indirect detection is typically much more promising than in supersymmetry for three reasons. First, there is no helicity suppression for the annihilation of bosonic KK dark matter into fermion pairs. Second, the preferred $B^{1}$ mass range is higher than in supersymmetry, leading to harder positron, photon, and neutrino spectra, with better signal-to-background ratio. And third, $B^{1}$ annihilation produces primary positrons and neutrinos with distinctive energy spectrum shapes, again facilitating observation above background. Kaluza-Klein gauge bosons therefore provide a promising and qualitatively new possibility for dark matter and dark matter searches.

[1] T. Kaluza, Sitzungsber. Preuss. Akad. Wiss. Berlin (Math. Phys. ) K1, 966 (1921); O. Klein, Z. Phys. 37, 895 (1926) [Surveys High Energ. Phys. 5, 241 (1986)].

[2] T. Appelquist, H.-C. Cheng and B. A. Dobrescu, Phys. Rev. D 64, 035002 (2001) hep-ph/0012100.

[3] K. R. Dienes, E. Dudas and T. Gherghetta, Nucl. Phys. B 537, 47 (1999) hep-ph/9806292.

[4] H.-C. Cheng, K. T. Matchev and M. Schmaltz, Phys. Rev. D 66, 036005 (2002) hep-ph/0204342.

[5] H.-C. Cheng, K. T. Matchev and M. Schmaltz, hep$\mathrm{ph} / 0205314$.

[6] G. Servant and T. M. Tait, hep-ph/0206071.

[7] See also E. W. Kolb and R. Slansky, Phys. Lett. B 135 , 378 (1984); J. Saito, Prog. Theor. Phys. 77, 322 (1987).

[8] M. W. Goodman and E. Witten, Phys. Rev. D 31, 3059 (1985).

[9] G. K. Mallot, Int. J. Mod. Phys. A 15S1, 521 (2000).

[10] J. R. Ellis, A. Ferstl and K. A. Olive, Phys. Lett. B 481, 304 (2000) hep-ph/0001005.

[11] M. Drees and M. Nojiri, Phys. Rev. D 48, 3483 (1993).

[12] M. Drees and M. M. Nojiri, Phys. Rev. D 47, 376 (1993).

[13] H.-C. Cheng, J. L. Feng and K. T. Matchev, in progress.

[14] N. J. Spooner et al. Phys. Lett. B 473, 330 (2000).

[15] A. Benoit et al., astro-ph/0206271.

[16] R. W. Schnee et al., Phys. Rept. 307, 283 (1998).

[17] H. V. Klapdor-Kleingrothaus, hep-ph/0104028.

[18] M. Bravin et al. [CRESST-Collaboration], Astropart. Phys. 12, 107 (1999) hep-ex/9904005.

[19] I. V. Moskalenko and A. W. Strong, Phys. Rev. D 60, 063003 (1999) astro-ph/9905283.

[20] J. L. Feng, K. T. Matchev and F. Wilczek, Phys. Rev. D 63, 045024 (2001) astro-ph/0008115.

[21] J. R. Ellis et al., Eur. Phys. J. C 24, 311 (2002).

[22] A. Barrau [AMS Collaboration], astro-ph/0103493.

[23] H. Hofer and M. Pohl, Nucl. Instrum. Meth. A 416, 59 (1998) hep-ex/9804016].

[24] L. Bergstrom, P. Ullio and J. H. Buckley, Astropart. Phys. 9, 137 (1998) astro-ph/9712318.

[25] H. F. Sadrozinski, Nucl. Instrum. Meth. A 466, 292 (2001).

[26] MAGIC Collaboration, M. Martinez et al., OG.4.3.08 in Proceedings of ICRC99, Utah, 17-25 August 1999. 